\title{
Sodium ferulate induces bone marrow mesenchymal stem cells to differentiate into neural cells by NF-kB signal pathway
}

\author{
Yuanlei Lou ${ }^{1}$, YangWang ${ }^{1}$, Xianliang Lai ${ }^{2}$, Zhifeng Deng ${ }^{2}$ \\ ${ }^{1}$ Institute of Urology, First Affiliated Hospital of Nanchang University; ${ }^{2}$ Department of Neurosurgery, the Second Affiliated \\ Hospital of Nanchang University, Nanchang 330006, China
}

Bone marrow stromal cells (MSCs) are nonhematopoietic stem cells in bone marrow, capable of self-renew and with pluripotency to differentiate into various cell types. A number of publications have proved that MSCs can be induced into progenitors of neural cells, neurons and glia by some inductors. In our previous study, we demonstrated that sodium ferulate (SF) could induce MSCs to differentiate into neural-like cells in vitro. However, the mechanism about the action of SF on inducing MSCs to undergo neurogenic differentiation is unclear. The present study was to explore whether the NF- $\kappa$ B signaling pathway contributes to the differentiation of rat MSCs (rMSCs) into neurallike cells induced by SF. rMSCs were cultured in the medium with $1 \mathrm{mg} / \mathrm{ml}$ of SF. The results of morphologia observation showed rMSCs change obviously from flat, elongated cells to spherical and refractile cells with several branching extensions, exhibiting a typical neuronsal morphology after treated with SF for $6 \mathrm{~h}$. The number of neurallike cells increased significantly with time and their processes formed extensive networks during the induction process. Immunofluorescence staining showed that rMSCs induced by SF gradually increased the expression of nestin, characteristic of neuronal precursor stem cells, as well as neuron-specific enolase (NSE), a marker of mature neurons, and glial fibrillary acidic protein (GFAP), the mature astrocyte marker. Immunofluorescence staining also showed that NF-kB/p65 protein was located in nuclear in a majority of rMSCs exposured to SF for 30 min, whereas $\mathrm{NF}-\kappa \mathrm{B} / \mathrm{p} 65$ protein was located in plasm in a majority of rMSCs cultured without SF. Western blot analysis also showed the expression of $\mathrm{NF}-\mathrm{kB} / \mathrm{p} 65$ in the nuclear was at high level in rMSCs treated with SF. Our data indicate that NF-KB signaling pathway may contribute to the differentiation of rMSCs into neural-like cells induced by SF.

Keywords: marrow stromal cell, sodium ferulate, neural cell

Cell Research (2008) 18:s147. doi: 10.1038/cr.2008.237; published online 4 August 2008

Correspondence: Zhifeng Deng

E-mail: dengzf63@126.com 